\title{
A Review Of Different Oropharyngeal Dysphagia Therapies By Speech And Language Pathologists
}

Ehsan Nadeifar ${ }^{1 *}$, Neda Tahmasebi Garmatani ${ }^{1}$

${ }^{1}$ Musculoskeletal Rehabilitation Research Center, Ahvaz Jundishapur University of Medical Sciences, Ahvaz, Iran.

*Corresponding Author: Email: e.naderi.slp@gmail.com

Background and aims: Oropharyngeal dysphagia is one of the common symptoms after stroke and is a marker for poor prognosis. Select the best therapy procedure is important. This study reviews the various therapy methods available to clinicians.

Methods: An electronic database search was performed on PubMed and Embase. The search was limited to English publications. Terms such as stroke, dysphagia, swallowing disorders; deglutition, deglutition disorders, treatment outcome, electro-stimulation-therapy, thermal-stimulation, muscletraining, rehabilitation, also extensive manual searching was conducted.

Results: oropharyngeal dysphagia interventions are divided into 5 groups based on the type of therapy: 1- bolus modifications and management (Compensatory Techniques), 2- swallow maneuvers and postures (Compensatory Techniques and/or Rehabilitative Techniques) 3- combination of interventions (Compensatory Techniques and/or Rehabilitative Techniques), 4- electro-stimulation and thermo-tactile stimulation (Facilitation Techniques), 5- other interventions (Rehabilitative Techniques).

Conclusion: There are different therapy procedures that can be use for Oropharyngeal dysphagia after stroke, but there are questions about the most effective of therapy, although some positive significant outcome studies have been published.

Key words: stroke, dysphagia, swallowing disorders, deglutition, electro-stimulation-therapy

DOI: 10.7575/aiac.abcmed.ca1.24

Published Date: February 2017

Peer-review is under responsibility of the 9th Iranian Stroke Congress.

Published by Australian International Academic Centre, Australia

This published work is open access under the CC BY license.

Available online at www.abcmed.aiac.org.au 